\title{
Questes
}

\section{Le doute : introduction}

\section{Servane Michel et Francesco Montorsi}

\section{(2) OpenEdition}

Journals

Édition électronique

URL : http://journals.openedition.org/questes/898

DOI : 10.4000/questes.898

ISSN : 2109-9472

\section{Éditeur}

Les Amis de Questes

\section{Édition imprimée}

Date de publication : 15 mars 2012

Pagination : 8-21

ISSN : 2102-7188

\section{Référence électronique}

Servane Michel et Francesco Montorsi, «Le doute : introduction », Questes [En ligne], 23 | 2012, mis en ligne le 01 janvier 2014, consulté le 22 septembre 2020. URL : http://journals.openedition.org/ questes/898; DOI : https://doi.org/10.4000/questes.898 


\section{Introduction}

\section{Servane MICHEL et Francesco MONTORSI}

Si le doute semble une notion éminemment moderne, il est indéniable que l'attitude qu'elle conceptualise existait bien avant Montaigne. Ce que la modernité s'est attachée à définir et dont elle a fait son emblème avait ses formes propres au Moyen Âge, qu'il s'agit d'explorer à travers textes, discours, pratiques. Il ne s'agit pas de chercher les prémices d'une attitude vouée à atteindre par la suite son épanouissement. Tout en restant conscients du biais que nous impose notre regard post-moderne, il nous revient de cerner les formes que prenait alors cette posture qui nous est familière. Étudier les formes du doute dans ce qui a pu être défini comme « un âge de la foi » ${ }^{1}$ s'avère une attitude féconde pour le chercheur : elle permet à la fois de mettre au jour des attitudes, des discours, des coutumes qu'il ne soupçonnait pas et, de ce fait, de révéler ce qui biaise habituellement notre regard sur le monde médiéval. L'un de ces facteurs est objectif et consiste dans la nature de nos sources: principalement cléricales, elles sont tributaires des conditions dans lesquelles elles ont été produites, et de l'usage qui en était prévu ${ }^{2}$. L'autre facteur est subjectif : bien souvent, à nos yeux, le Moyen Âge représente

\footnotetext{
${ }^{1}$ Sabina Flanagan, Doubt in an Age of Faith: Uncertainty in the Long Twelfth Century, Turnhout, Brepols, «Disputatio», 17, 2008, p. 2-3. Voir également, du même auteur, «Lexicographic and Syntactic Explorations of Doubt in Twelfth-Century Latin Texts », Journal of Medieval History, 27 (2001), p. 219-240.

2 Alexander Murray souligne à ce sujet que «les clercs qui mirent par écrit la plus grande partie de cette masse de [preuves] étaient loin d'avoir pour tâche de consigner scrupuleusement les croyances religieuses de leur monde pour notre profit : il existe un élément de propagande dans une grande partie de ce qu'ils ont écrit. Si l'on se représente, en même temps, qu'il existe très peu de preuves, même à l'intérieur de ces sources, des croyances religieuses en vigueur dans la population, il faut reconnaître que l'expression "âge de la foi" repose sur de bien faibles bases » (Alexander MuRRAY, Reason and Society in the Middle Ages, Oxford, Clarendon Press, 1978, p. 6. Nous traduisons).
} 
une parenthèse de foi entre le scepticisme antique - oublié après l'Antiquité et redécouvert au $\mathrm{XVI}^{\mathrm{e}}$ siècle - et le scepticisme moderne de la Renaissance qui conduira plus tard au doute philosophique de Descartes ${ }^{3}$. Ainsi, entre Pyrrhon et Montaigne, on s'est pendant longtemps limité à tenir pour acquis un silence du doute, pour ensuite constater son «irruption» avec la modernité ${ }^{4}$. L'image même qu'on se fait de la modernité occidentale semble en partie reposer sur la naissance de ce doute post-médiéval, qui favorise la conceptualisation historique et le compartimentage chronologique. Ne nous sentons-nous pas les descendants du «Que sais-je ?» de Montaigne, ou de ce François Rabelais qui, couché sur son lit de mort, mande à dire au cardinal Du Bellay « Je vais chercher un grand peut-être »? La Renaissance serait la rupture. Avec elle, c'est l'irruption du doute. Et, par là, de la modernité.

Les limites de cette approche sont évidentes et, depuis des années, la critique a enquêté, avec profit, sur ce doute qui assaillait l'homme du Moyen Âge, et qui est surtout, à nos yeux, un élément intrinsèque de la pensée rationnelle ${ }^{5}$. Si les études scientifiques ont tardé à élucider cet aspect pour le Moyen Âge, c'est peut-être dû à la force d'un malentendu. Les siècles précédant la Renaissance ont répété avec force et sans cesse

\footnotetext{
${ }^{3}$ Alexander Murray compare à celle de l'âge d'or l'image que nous nous faisons de la foi médiévale, qui « remplit une fonction psychologique pour nous : elle est ou bien la base d'une critique nostalgique de l'irréligion actuelle, ou, en sens opposé, un moyen de désavouer du même coup le passé et la religion »(Alexander MurRAY, Reason and Society in the Middle Ages, op. cit., p. 6. Nous traduisons).

${ }^{4}$ L' "Irruption du doute » est le titre de la cinquième partie de l'ouvrage de Thierry HENTSCH, Raconter et mourir. L'Occident et ses grands récits, Rosny-sous-Bois, Bréal, 2002, p. 327-415. On y trouve des chapitres consacrés à Rabelais, Cervantès, Shakespeare et Descartes.

${ }^{5}$ Ces dernières années, le doute philosophique médiéval a été le sujet de plusieurs recherches. Cf. Dominik PERLER, Zweifel und Gewissheit. Skeptische Debatten im Mittelalter, Francfort-sur-le-Main, Vittorio Klostermann, 2006 ; Sabina FlAnAGAN, «Lexicographic and Syntactic Explorations of Doubt...», art. cit.; Peter DINZELBACHER, Unglaube im «Zeitalter des Glaubens ». Atheismus und Skeptizismus im Mittelalter, Badenweiler, Wissenschaftlicher Verlag Bachmann, 2009 ; Rethinking the History of Skepticism. The Missing Medieval Background, Henrik LAGERLUND (dir.), Leyde/Boston, E. J. Brill, 2010.
} 
l'idée qu'un certain type de doute était interdit. Il s'agit du doute blasphématoire - prémisse de l'incrédulité - portant sur la personne du Sauveur, doute exprimé, entre autres, dans le fameux verset de l'Évangile «Modice fidei, quare dubitasti ? ${ }^{6}$. Des siècles plus tard, Jean de Salisbury exprime avec fermeté la profonde aversion médiévale pour le doute concernant les vérités de la foi : "Qui vero an Deus sit deducit in quaestionem et an idem potens, sapiens sit, an bonus, non modo irreligiosus sed perfidus est $\gg^{7}$.

Mais, si l'on fait abstraction de l'incrédulité, péché sans possibilité de rémission, le doute, au Moyen Âge, est toujours admis et souvent bénéfique. Chez un grand nombre de penseurs il est considéré comme le début de la connaissance, la première impulsion vers la compréhension du vrai. Dante exprimera cette conviction avec une belle image poétique : «A guise de surgeon / Le doute alors au pied du vrai provigne ; / De puy en puy nature au som nous pousse $»^{8}$.

\section{Étymologie}

Le doute au Moyen Âge a aussi une résonance différente de celle qu'il a aujourd'hui. Pour essayer de la saisir, on peut recourir à la pratique médiévale de l'étymologie. Dans son encyclopédie, Isidore de Séville donne l'étymologie correcte du mot dubius : «Dubius, incertus; quasi

\footnotetext{
${ }^{6}$ «Homme de peu de foi, pourquoi as-tu douté ?» (Matthieu, XIV, 31).

${ }^{7}$ «Celui qui met en doute que Dieu soit puissant, savant et bon, non seulement il est sans foi, mais il est aussi perfide» (Jean DE SALisbury, Policraticus sive De nugis curialium et vestigiis philosophorum, Clemens C. I. WEBB (éd.), Oxford, 1909, livre VII, ch. 8. Nous traduisons).

8 DANTE, Euvres complètes, André PÉZARD (éd. et trad.), Paris, Gallimard, 1965, «Bibliothèque de la Pléiade », 182, p. 1393, v. 130-132. Dans la note relative au v. 131, André Pézard glose: «Le doute: entendre ici "le besoin de savoir des vérités nouvelles" ». Le mot italien est, bien entendu, « dubbio ».
} 
duarum viarum ${ }^{9}$. L'adjectif dubius et le verbe dubitare dérivent en effet, comme le savait Isidore, de la racine duo, «deux » (nous retrouvons cela aussi en allemand, où le mot zweifeln garde la trace du lien avec zwei, « deux »). Par conséquent, le mot dubitare indique d'abord l'idée de l'hésitation entre deux choses, qu'il s'agisse de deux pensées ou de deux actions. Douter c'est, selon cette image, se trouver devant une bifurcation (« quasi duarum viarum »).

Déjà en latin tardif, un autre sens s'était greffé sur cette acception. Le sentiment d'insécurité et d'incertitude lié à l'hésitation aboutissent au développement du sens de «craindre $»^{10}$. En effet, la première attestation du mot de douter en ancien français a ce sens que le mot perdra ensuite, mais qui perdure dans le verbe redouter, lequel est à l'origine un intensif de douter. Au cours du Moyen Âge, l'acception «craindre » est prédominante par rapport à celles de «hésiter», «être dans l'incertitude» et, bien souvent, il n'est pas aisé de distinguer les deux sens ${ }^{11}$, comme on peut le voir dans certains exemples commentés dans ce bulletin. Il est intéressant de voir que la dernière attestation du sens «craindre » date du début du $\mathrm{XVII}^{\mathrm{e}}$ siècle. Étrange coïncidence : à la même époque, Descartes invente une formule vouée à une brillante carrière, le doute philosophique, alors que, quelques années auparavant (en 1580), Montaigne avait le premier utilisé le verbe avec le sens de «n'être sûr de rien, professer le scepticisme $»:\left\langle\right.$ je doute $»^{12}$.

Ce survol étymologique nous montre les échos que le «doute» suscitait en ancien français et nous donne la mesure du clivage qui nous

\footnotetext{
9 «Qui hésite, qui est incertain. Comme devant une bifurcation » (ISIDORE DE SÉvILLE, Etimologie o origini, Angelo Valastro Canale (éd.), Turin, Utet, 2004, 1, X, 77, p. 810. Nous traduisons).

${ }^{10}$ Cf. Französisches Etymologisches Wörterbuch, Walther von WARTBURG (dir.), Tübingen, J.C.B. Mohr, 1949, III, entrée « douter », p. 169-170.

${ }^{11}$ Ibid., p. 170.

12 Michel de Montaigne, Les Essais, Pierre Villey (éd.), Paris, PUF, 1992, 3 vol., vol. 1, livre II, chap. XII « Apologie de Raimond Sebond », p. 502 et 527.
} 
sépare de l'ancienne conception. En ancien et moyen français, le mot avait une gamme de sens plus vaste qu'aujourd'hui. D'abord, il gardait le premier sens de l'étymon latin, «hésiter », qui aujourd'hui n'est plus perçu avec netteté. Mais surtout, douter avait une plus grande intensité émotionnelle en ancien français, car il était intrinsèquement lié au sentiment de la peur. Les évolutions philosophiques de la conceptualisation du doute, bien montrées par Montaigne et Descartes, ont fini par évacuer le sens inquiétant du doute, celui lié à l'affectivité, au profit d'une vision plus philosophique, qui, en fin de compte, est celle qui prévaut dans le sens actuel.

Les différents sens de douter au Moyen Âge se retrouvent dans la définition du mot dubitare que Pierre Bersuire donne dans le Repertorium morale (rédigé entre 1335 et 1342), qui nous explique qu'il y a trois types de doute, celui des savants, celui des faibles, celui des désespérés :

Constat enim quod est dubitatio Sapientum, Imbecillium, Desperantium. Sapientes nihil temere volunt afferere, sed potius volunt negocia prudenter sub quodam dubio palliare. Et de hoc dicit quod dubitare de singulis non est inutile. Imbecilles vero solent, ubi timor non est, trepidare, et nunquam securi esse, sed in omnibus dubitare [...]. Desperantes etiam solent dubitare de salute aeterna, non sperare, propter quam solent ad mala omnia se laxare. (Matth 28). Quidam autem dubitaverunt. ${ }^{13}$

\footnotetext{
${ }^{13}$ «Il est clair qu'il y a le doute des savants, le doute des faibles d'esprits, le doute de ceux qui désespèrent. Les savants ne veulent pas argumenter au hasard, ils veulent plutôt prudemment envelopper leurs pensées par un certain doute. Et pour cela, on dit qu'il n'est pas inutile de douter de chaque chose. Les faibles d'esprit ont coutume de trembler là où la crainte n'a pas raison d'être, de ne jamais être sûrs et de douter de toute chose. [...] Ceux qui désespèrent doutent du salut éternel et pour cette raison ils s'abandonnent à tous les maux. Matthieu, XXVIII, "quelques-uns eurent des doutes" » (Pierre BERSUIRE, Dictionarii seu Repertorii moralis Petri Berchorii Pictaviensis Pars prima (-Pars tertia), Venetis, Apud Haeredem Hyeronymi Scoti, 1583, 3 tomes, t. I, p. 512. Nous traduisons).
} 


\section{Peur, hésitation}

Un certain nombre des aspects évoqués par Bersuire seront abordés dans les différentes contributions de ce bulletin, mais le domaine relatif au doute médiéval est trop vaste pour être épuisé ici. Ainsi qu'on vient de le voir, le réseau sémantique et conceptuel lié au doute ne se superpose pas avec le nôtre. En effet, le doute, au Moyen Âge, est un état d'esprit qui est associé à d'autres sentiments, notamment la peur et l'indécision. Une analyse du doute médiéval doit, nous semble-t-il, tenir compte aussi de cette réalité.

Le doute n'est pas séparable de la crainte et de l'hésitation. Si on ne trouve pas d'interventions concernant spécifiquement la crainte, par contre un certain nombre d'exemples du doute-hésitation peuvent être retrouvés dans les contributions à ce bulletin. En effet, l'hésitation a une place prépondérante au théâtre (que l'on pense à l'hésitant par excellence qu'est Hamlet - une autre figure de la modernité), spécialement en relation avec l'artifice du monologue, où souvent le personnage révèle au public ses dilemmes. Mais la littérature elle aussi présente un certain nombre d'hésitations. La plus célèbre est sans doute celle de Lancelot devant la charrette infamante. Les « deux pas » qu'il « demore» sont une hésitation suffisante pour déclencher, par la suite, les remontrances de Guenièvre qui reprochera au chevalier sa peur et son hésitation (les deux sens étant ici contigus) :

«Comant? Dont n'eüstes vos honte

De la charrete et si dotastes?

Molt a grant enviz i montastes

Quant vos demorastes deus pas. » ${ }^{14}$

${ }^{14}$ Chrétien de Troyes, Le Chevalier de la Charrette, Alfred Foulet et Karl David UitTi (éd.), Paris, Garnier, 2010, v. 347-368 et v. 4502-4503. Ces deux derniers vers ne se retrouvent pas dans la copie de Guiot, et par conséquent on ne peut pas les lire malgré leur importance pour la compréhension du passage - dans plusieurs éditions modernes du texte. Ils ont été le prétexte d'une controverse, qui a porté sur la manière d'éditer Chrétien de Troyes : cf. Eugène VinAVER, «Les Deux Pas de Lancelot», in 
Si le doute-hésitation est bien attesté par les témoignages littéraires, le doute-crainte est, si l'on veut, consubstantiel à la lyrique médiévale, car l'amour est, bien entendu, l'un des hauts lieux de la peur. C'est une vérité aussi vieille que le monde, les amoureux sont craintifs et les littératures de tous les pays et de toutes les époques en gardent des témoignages. Parmi les plus belles réussites dans la représentation littéraire de cette «peur d'amour », on trouve l'une des perles de la littérature grecque, le fragment de la poétesse Sappho de l'Ode à une femme aimée. De même, Ovide dira plus tard, dans des vers célèbres, que, quand on est amoureux, on craint toute chose : «cuncta timemus amantes $»^{15}$. La poésie d'amour du Moyen Âge accepte, intègre et peut-être développe encore davantage cette association universelle entre l'amour et la peur. En effet, la crainte et l'hésitation sont des composantes fondamentales de l'amour courtois et l'élan mystique vers la dame est toujours contrebalancé par la peur du refus ou par 1'hésitation qui paralyse ${ }^{16}$. Ainsi, Bernard de Ventadour pourra dire que la fin' amor ne peut pas exister sans la « paor» et la «doptansa » :

Mas greu veiretz fin' amansa

ses paor e ses doptansa

c'ades tem om vas so c'ama, falhir,

per qu'eu no.m aus de parlar enardir. ${ }^{17}$

Mélanges Jean Fourquet, Paul VALENTIN et Georges ZINK (éd.), Munich/Paris, Hüber/Klincksieck, 1969, p. 335-351 ; Karl David UITTI, «On editing Chrétien de Troyes : Lancelot's Two Steps and their Context», Speculum, LXIII (1988), p. 271-292 ; David F. Hult, «Steps Forward and Their Context : More on Chrétien's Lancelot », Speculum, LXIV (1989), p. 307-316.

${ }^{15}$ OvIDE, Métamorphoses, lib. VII, v. 719.

${ }^{16}$ Des réflexions concernant le lexique de la peur dans la lyrique médiévale française se trouvent dans Georges LAVIS, L'Expression de l'affectivité dans la poésie lyrique française du Moyen Âge (XII ${ }^{e}-X I I I^{e}$ siècles). Étude sémantique et stylistique du réseau lexical joie-dolor, Paris, Les Belles Lettres, 1972, p. 94-113 et dans Glynnis M. CROPP, Le Vocabulaire courtois des troubadours de l'époque classique, Genève, Droz, 1975, «PRF », 135, p. 200-203.

17 «Mais vous verrez difficilement un amour parfait, sans peur ni frayeur, puisque l'on craint toujours de commettre une faute à l'égard de celle que l'on aime ; je n'ai donc pas le courage d'oser parler» (BERNARD DE VENTADOUR, Ab joi mou lo vers e.l comens, v. 13-16, cité et traduit par Glynnis M. CROPP, Le Vocabulaire courtois..., op. cit., 


\section{Le doute philosophique : possibilité et critique d'un scepticisme médiéval}

Un examen des discussions médiévales sur le doute révèle le biais par lequel ce dernier est toujours abordé : la tournure d'esprit des penseurs médiévaux les porte plus à expliquer le phénomène de la croyance - c'està-dire de la foi - qu'à adopter une perspective purement épistémologique et profane. L’épistémologie de la croyance se développe cependant avec rigueur et précision, soulevant la question de la distinction à établir entre foi et connaissance ${ }^{18}$.

Cependant une approche non-religieuse du doute prévaut chez Jean de Salisbury, dont la pensée est considérée comme la plus proche de l'attitude moderne en matière de doute ${ }^{19}$. Si c'est un fait que le Moyen Âge

p. 200). On peut lire ce poème dans plusieurs éditions et anthologies. Nous signalons Los trovadores. Historia literaria y textos, Martín DE RIQUER (éd.), Barcelona, Ariel, 1992, 3 vol., vol. 1, p. 392.

${ }^{18}$ Pour une analyse comparée des données du débat, voir Sabina Flanagan, Doubt in an Age of Faith..., op. cit., ch. 4, p. 91-155. Elle expose les réflexions de Baldwin de Forde (De Commendatione Fidei), selon qui la foi peut être définie comme une connaissance, d'Abélard (Theologia 'scholarium') et d'Hugues de Saint-Victor (De Sacramentis) - chez lequel elle discerne une forme d'analyse du langage ordinaire ( "common language" methodology»). Le point commun de ces penseurs est la position suivante : puisque la foi est un type de certitude - quelle que soit sa relation avec la connaissance - alors le doute n'y a pas sa place. De plus, là où le doute peut admettre plusieurs degrés, la foi ne le peut pas. Foi et doute ne sont donc pas des contraires - ce que montre, commente Sabina Flanagan, leur situation respective sur le continuum du doute.

19 Cf. JEAN DE SAlisbUry, Policraticus sive De nugis curialium et vestigiis philosophorum, éd. cit., lib. VII, ch. 2, II, 98-99 : « sunt autem dubitabilia sapienti quae nec fidei nec sensus aut rationis manifestae persuadet auctoritas et quae suis in utramque nituntur firmamentis » ( «Il existe cependant des objets de doute pour le sage, que ne résout l'autorité ni de la foi, ni des sens ou de la raison manifeste et dont chacune des deux explications possibles s'appuie sur une preuve solide ». Nous traduisons). Parmi ces questions se trouvent celles «que l'on peut poser en toute piété au sujet de Dieu lui-même qui dépasse la capacité d'investigation de toute nature rationnelle » («quae pie quaeruntur de ipso Deo qui totius naturae rationalis excedit inuestigationem ». Nous traduisons). Cependant, mettre en doute l'existence de Dieu reste irréligieux, et même «perfidus » (ch. 3). 
n'a pas connu de courant sceptique identifiable comme tel ${ }^{20}$, Jean de Salisbury reste un exemple de philosophe ayant développé des arguments de type sceptique ${ }^{21}$. Chez lui et chez les autres penseurs ayant interrogé les arguments hérités de l'Académie, le scepticisme n'est pas vécu ni même pensé sur un mode éthique ou comme conception du monde-à la différence du pyrrhonisme des Anciens. Les réflexions sceptiques ne visent nullement l'épochê - la suspension du jugement chère aux philosophes antiques - ni la libération par rapport à l'inquiétude philosophique. En effet, la certitude donnée par la foi domine attitudes et modes de pensée, de sorte que le doute n'apparait que comme un outil épistémologique.

Les représentants du scepticisme médiéval ont en commun une mise en question de la confiance aristotélicienne en la raison, laquelle, dans la plupart de leurs conclusions, est reconnue incapable de correspondre par ses propres moyens à l'incommensurabilité des vérités éternelles révélées par Dieu. Leur utilisation des arguments sceptiques est difficile à rattacher à la tradition pyrrhonienne, peu connue à l'époque médiévale et surtout biaisée par la présentation qu'en donne Augustin, principale source pour la connaissance de la philosophie antique mais adversaire farouche du scepticisme $^{22}$. Les développements médiévaux sur l'argumentation

\footnotetext{
${ }^{20}$ Voir Charles BoYARD, entrée «Medieval Skepticism », in Edward N. ZALTA (éd.), The Stanford Encyclopedia of Philosophy (Winter 2010 Edition), http://plato.stanford.edu/archives/win2010/entries/skepticism-medieval/. Une abondante bibliographie donne les sources médiévales et les travaux critiques les plus récents.

${ }^{21}$ Le possible scepticisme de Jean de Salisbury est actuellement étudié par Christophe GRELLARD (Université de Paris I), qui prépare un ouvrage sur Le Problème sceptique au Moyen Âge, de saint Augustin à Jean Buridan; il entend y montrer - à la suite de ses articles déjà parus-comment Jean de Salisbury ne se contente pas d'un doute épistémologique mais étend le scepticisme au domaine éthique, considérant la philosophie comme un mode de vie et faisant ouvertement profession de scepticisme. Voir aussi Christophe GRELlARD «Comment peut-on se fier à l'expérience ? Esquisse d'une typologie des réponses médiévales au scepticisme », Quaestio, 4 (2004), p. 113-135 et «Jean de Salisbury. Un cas médiéval de scepticisme », Freiburg Zeitschrift für Theologie und Philosophie, 54 (2007), p. 16-40.

${ }^{22}$ Le courant pyrrhonien du scepticisme fut pratiquement ignoré jusqu'à la fin du XIII ${ }^{\mathrm{e}}$ siècle: les œuvres de Sextus Empiricus, comme celles de Diogène Laërce,
} 
sceptique sont surtout connus grâce à leur condamnation par la Sorbonne en $1277^{23}$. À partir du XIV ${ }^{\mathrm{e}}$ siècle, les théories s'apparentant à des positions sceptiques, comme celle de la double vérité ${ }^{24}$ ou, plus largement, le doute porté sur la garantie divine de la connaissance, s'inscrivent dans le mouvement qui, à la fin du Moyen Âge, voit un recentrement de la pensée sur la perspective individuelle et une promotion de la subjectivité ${ }^{25}$. La réflexion épistémologique prend ses distances par rapport à la caution surnaturelle de la foi et s'autonomise à l'égard de la théologie. Cependant, que ce soit prudence face à l'autorité ecclésiastique ou réaffirmation de la foi personnelle, les penseurs qui développent les arguments sceptiques posent au seuil de toute réflexion philosophique la distinction entre la certitude propre à la foi - non soumise au doute - et la certitude issue de l'évidence naturelle, seul objet de la rationalité naturelle. Par ailleurs ironie de l'histoire - le doute est à ce point formalisé comme une méthode heuristique qu'il ne portera sur les questions de fond que lorsque les réponses à celles-ci auront été formulées. Comme le souligne Sabina Flanagan, c'est seulement «une fois que le nouveau matériel

connaissaient une très faible diffusion. On relève une connaissance partielle de la philosophie sceptique grecque chez Bède, Raban Maure et dans l'Orient byzantin et musulman. En revanche, le scepticisme académique avait été transmis, notamment par le commentaire d'Augustin aux Académiques de Cicéron (le Contra Academicos en est une réfutation).

${ }^{23}$ La Faculté de Théologie de Paris, sous l'autorité d'Étienne Tempier et du conseil de l'Université, condamna alors 219 thèses jugées inacceptables en philosophie et théologie et donc exclues de l'enseignement universitaire. Voir Roland HISSETTE, Enquête sur les 219 articles condamnés à Paris le 7 mars 1277, Louvain/Paris, Publications universitaires/Vander-Oyez, «Philosophes médiévaux », 22, 1977.

24 Il s'agit d'une théorie qui soutient que la raison peut démontrer des vérités contradictoires avec celles établies par la Révélation. Cependant, sa plus complète élaboration est celle présentée par la censure de 1277 et par les réfutations de Thomas d'Aquin. De la sorte, il est difficile de savoir si cette théorie a réellement été professée ou si elle a été constituée comme théorie seulement par les censeurs de la Sorbonne. Elle pourrait être illustrée par des philosophes comme Nicolas d'Autrecourt ou Jean Buridan, au XIV ${ }^{\mathrm{e}}$ siècle, qui admettent la possibilité de soutenir des thèses «probables »-de probabilis, «démontrable »-en désaccord avec les vérités de la foi.

${ }^{25}$ Cf. L'Individu au Moyen Âge. Individualisation et individuation avant la modernité, Brigitte Bedos-ReZaK et Dominique Iogna-Prat (dir.), Paris, Aubier, 2005. 
[aristotélicien] eut été suffisamment domestiqué que de telles questions furent autorisées à être débattues $»^{26}$.

Étant sauve la vérité de la Révélation divine, sur laquelle ne porte pas le doute, le questionnement vise les possibilités proprement humaines de la connaissance naturelle ${ }^{27}$. Demeure cependant l'héritage augustinien, qui réfute le scepticisme absolu tout en reprenant le doute comme point de départ épistémologique. La portée du doute est donc d'emblée réduite, chez les penseurs médiévaux, au domaine propre de la connaissance naturelle. Sous la plume des plus exigeants et rigoureux des philosophes, un scepticisme absolu, qui s'étendrait à toute forme de connaissance et de relation au monde, est à rejeter comme signe d'un manque de réflexion critique $^{28}$. L'exigence de rationalité que manifeste un Duns $\operatorname{Scot}^{29}$, par exemple, se traduit par le doute épistémologique : mettant en question les prétentions de la raison, il renonce à élaborer une théorie de la connaissance et cherche plutôt à établir fermement les conditions de possibilité de la certitude épistémologique. Son analyse métaphysique des structures de la connaissance humaine le conduit ainsi à réduire d'abord la certitude proprement rationnelle à quelques principes logiques évidents. Son rejet, cependant, d'un scepticisme absolu, qui nierait la possibilité même de toute connaissance, reste une posture a priori. La réflexion de Duns Scot est caractéristique de cette auto-limitation de la pensée médiévale au doute épistémologique, sans préjudice des certitudes d'une

\footnotetext{
26 «Once the new material had been sufficiently domesticated such questions were allowed to be debated» (Sabina Flanagan, Doubt in an Age of Faith..., op. cit., p. 155).

${ }^{27}$ Naturel doit ici s'entendre en opposition avec surnaturel, "guidé par une révélation divine ».

${ }^{28}$ C'est ce paradoxe que développe Lambertus Marie DE RIJK, La Philosophie au Moyen Âge, Leyde, E. J. Brill, 1985, p. 215.

29 Duns SCOT (1266-1308) est un frère mineur de l'ordre franciscain, défenseur notamment de l'immaculée conception de Marie. Sa béatification par l'Église catholique est le signe d'une reconnaissance par l'institution religieuse d'une pensée dont les exigences proprement rationnelles ne mettent pas en danger la foi.
} 
autre nature - comme celle apportée par l'expérience. De manière générale, l'usage que les penseurs médiévaux font du doute rationnel ne leur fait pas emprunter les mêmes voies que leurs prédécesseurs antiques. Le scepticisme médiéval est constitué d'une série de préoccupations et de réponses apportées aux problèmes sceptiques. Plutôt que de scepticisme, Lambertus Marie de Rijk propose donc de parler d'un « criticisme radical », qui limite toutes les certitudes apparentes pour donner plus de poids aux certitudes réelles - celles garanties par la Révélation d'un Dieu dont la rationalité est plus fiable que celle de l'esprit humain. Les théories critiques les plus extrêmes constituent une opposition entre vérité et probabilité, entre vérité et évidence naturelle. La Révélation divine, d'emblée reçue comme vraie et, partant, non soumise au doute, bouleverse la raison naturelle qui se voit alors poser des limites. Le doute porte alors non pas sur la possibilité de la connaissance mais sur les conditions de possibilités de cette connaissance - ou mieux, sur la mesure de cette connaissance humaine, eu égard à l'inaccessibilité des vérités suprêmes à la raison naturelle.

\section{Théâtre et théologie : le doute intégré}

Le théâtre, forme libre s'il en est au Moyen Âge, offre un terrain privilégié à l'expression littéraire et populaire du doute. À l'intérieur du vaste champ étudié sous l'image englobante du carnaval ${ }^{30}$, les figures de la folie ${ }^{31}$, de l'étrange, de l'autre, offrent au chercheur moderne des formes originales d'expression du doute à l'intérieur d'une société d'ordre et d'une

\footnotetext{
${ }^{30}$ L'œuvre de Mikhaïl Bakhtine à ce sujet reste décisive (Mikhaïl BAKHTINE, L'CEuvre de François Rabelais et la culture populaire au Moyen Âge et sous la Renaissance, Andrée RoBEl (trad .), Paris, Gallimard, «Bibliothèque des idées », 68, 1970 [1 ${ }^{\text {ère }}$ éd. russe 1965]).

31 Voir à ce sujet Jean-Marie FrITZ, Le Discours du Fou au Moyen Âge, Grenoble, Presses Universitaires de Grenoble, 1992 (en particulier le chapitre XV sur la théâtralité).
} 
culture fondée sur la foi. Le Jeu de la Fenillée d'Adam de la Halle est à cet égard l'une des œuvres les plus étranges et insaisissables que le Moyen Âge oppose à notre regard moderne, au rationalisme qui prévaut jusque dans les études littéraires. Antérieures à l'âge des règles et de la bienséance, les pièces médiévales ouvrent à l'expression populaireparfois jusque dans les sanctuaires - un champ de liberté qui, même sous le contrôle du clergé, atteste de l'acceptation du doute dans l'espace public et sacré.

Les mystères-étudiés dans deux des communications de ce bulletin - illustrent les paradoxes d'une foi qui se proclame et se met en question dans la double énonciation de la scène théâtrale. La figure de l'apôtre Thomas pourrait résumer à elle seule les attitudes contrastées qu'adopte l'homme médiéval en proie au doute. Férus d'étymologie, les théologiens médiévaux (Bède le Vénérable, Théophylacte d'Odred) ont parfois interprété la personnalité de Thomas à partir de son nom - qui en araméen signifie «le double», ou «le jumeau»-pour expliquer son hésitation, son oscillation entre un doute qui fait de lui un personnage dur et une foi aussi soudaine que sublime qui l'élève au dessus des autres apôtres. Le théâtre devait donner à ces spéculations une incarnation saisissante, à en croire les pièces anglaises qui nous sont conservées ${ }^{32}$ : elles font monter sur la scène un personnage solitaire et tourmenté, préfiguration d'Hamlet qui donne à entendre, dans un long monologue introspectif, les doutes qui l'assaillent et l'isolent du groupe des apôtres. À travers l'apôtre du doute, le Moyen Âge s'offre sous plusieurs visages. Iconographie, théologie, art dramatique laissent planer sur lui une ombre -

${ }^{32}$ Cf. Ludus Coventriae, or The plaie called Corpus Christi, Katherine SALTER BlocK (éd.) Londres, Oxford University Press, «Early English Texts Society », 120, 1922 ; Thomas of India, in The Wakefield mystery plays, Martial RosE (éd.), New York, Doubleday, 1962. 
«the ghost», selon le mot d'Alexander Murray $^{33}$ - qui semble être ce «péché originel» du doute. Les fluctuations de fortune qu'a connu le personnage de Thomas au cours du Moyen Âge soulignent la méfiance fondamentale que nourrit «l'âge de la foi » envers tout ce qui ébranle les fondements de sa pensée et de sa conception du monde. Cependant, les justifications théologiques du doute de Thomas témoignent d'un effort de rationalisation qui a poussé les théologiens à justifier une attitude que leur foi, notamment celle enseignée par les Pères de l'Église, ces autorités indépassables, considérait comme inacceptable. C'est enfin l'évolution ultime de la piété chrétienne aux $\mathrm{XIV}^{\mathrm{e}}$ et $\mathrm{XV}^{\mathrm{e}}$ siècles qui a redonné au doute une valeur, en en faisant l'une des formes du désir de Dieu.

\footnotetext{
${ }^{33}$ Alexander Murray, Doubting Thomas in Medieval Exegesis and Art, Rome, Unione Internazionale degli istituti di archeologia, storia e storia dell'arte in Roma, 2006, p. 45 et $s q q$.
} 